\title{
AN ANALOGUE OF THE OPERATOR CURL FOR NONABELIAN GAUGE GROUPS AND SCATTERING THEORY
}

\author{
A. SEVOSTYANOV
}

\begin{abstract}
We introduce a new perturbation for the operator curl related to connections with nonabelian gauge groups over $\mathbb{R}^{3}$. We also prove that the perturbed operator is unitary equivalent to the operator curl if the corresponding connection is close enough to the trivial one with respect to a certain topology on the space of connections.
\end{abstract}

\section{INTRODUCTION}

It is well known that the operator curl appears in various fields of mathematics and physics. Although its spectral theory was developed quite recently (see 4, 10] and references there). Interesting spectral problems for the operator curl appear when it is defined in nontrivial regions of the Euclidean space $\mathbb{R}^{3}$.

As usual, another interesting class of spectral problems for the operator curl would appear if we were be able to add a perturbation to it in such a way that the perturbed operator is close, in some sense, to the original one. A natural operator $\operatorname{curl}_{A}$ with potential $A$ that generalizes the operator curl in differential geometry is the composition of the Hodge star operator and the covariant derivative associated to a connection $A$ with a nonabelian gauge group and defined over $\mathbb{R}^{3}$ (see formula (6) below). But from the point of view of spectral theory that operator is quite different from curl. For instance, the scattering theory for the pair $\left(\operatorname{curl}_{A}, \operatorname{curl}_{0}\right)$ can not be properly developed since both operators are not elliptic.

In this paper we introduce another operator $X_{A}$ related to nonabelian gauge groups. The operator $X_{A}$ is not a differential operator (see formula (5)). In fact, to define it one should also consider an analogue of the divergence operator div for noncommutative gauge groups (see formula (2)). The operator div is naturally related to the operator curl since the kernel of div is the natural domain where the operator curl becomes elliptic.

The spectral properties of the operator $X_{A}$ introduced in this paper are similar to those of curl. In particular, we prove that if the connection $A$ is small in some sense then the operator $X_{A}$ is unitary equivalent to $X_{0}$. Technically the solution to this problem is achieved by extending $X_{A}$ to an elliptic operator that acts in a bigger space. This trick is similar to that used in [2, 3] for the Maxwell operator.

Acknowledgments. The author thanks Prof. M. Sh. Birman for useful advises. I would like also to express my gratitude to N. Filonov and A. Pushnitski. The analytic details of this paper would never be completed without their support.

2000 Mathematics Subject Classification 47A40 Primary; 35P25 Secondary Key words and phrases. Selfadjoint operator, Scattering theory. 


\section{Setup}

Let $K$ be a compact semisimple Lie group, $\mathfrak{k}$ its Lie algebra and $\mathfrak{g}$ the complexification of $\mathfrak{k}$. We denote by $(\cdot, \cdot)$ the Killing form of $\mathfrak{g}$. Recall that the restriction of this form to $\mathfrak{k}$ is nondegenerate and negatively defined.

Let $\Omega^{*}\left(\mathbb{R}^{3}, \mathfrak{k}\right)$ be the space of smooth $\mathfrak{k}$-valued differential forms on $\mathbb{R}^{3}$, and $\Omega_{c}^{*}\left(\mathbb{R}^{3}, \mathfrak{k}\right)$ the space of smooth $\mathfrak{k}$-valued differential forms on $\mathbb{R}^{3}$ with compact support. We define a scalar product on $\Omega_{c}^{*}\left(\mathbb{R}^{3}, \mathfrak{k}\right)$ by

$$
<\omega_{1}, \omega_{2}>=-\int_{\mathbb{R}^{3}}\left(\omega_{1} \wedge, * \omega_{2}\right)=-\int_{\mathbb{R}^{3}} *\left(\omega_{1} \wedge, * \omega_{2}\right) d^{3} x, \omega_{1,2} \in \Omega_{c}^{*}\left(\mathbb{R}^{3}, \mathfrak{k}\right)
$$

where $*$ stands for the Hodge star operation associated to the standard Euclidean metric on $\mathbb{R}^{3}$, and we evaluate the Killing form on the values of $\omega_{1}$ and $* \omega_{2}$ and also take their exterior product.

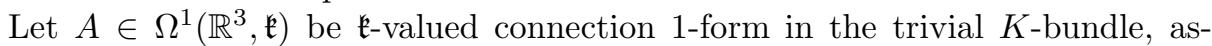
sociated to the adjoint representation of $K$, over $\mathbb{R}^{3}, F \in \Omega^{1}\left(\mathbb{R}^{2}, \mathfrak{k}\right)$ its curvature 2 -form, $F=d A+\frac{1}{2}[A \wedge A]$. Here as usual we denote by $[A \wedge A]$ the operation which takes the exterior product of $\mathfrak{k}$-valued 1 -forms and the commutator of their values in $\mathfrak{k}$, and $d$ stands for the exterior derivative.

We recall that the covariant derivative $d_{A}: \Omega_{c}^{n}\left(\mathbb{R}^{3}, \mathfrak{k}\right) \rightarrow \Omega_{c}^{n+1}\left(\mathbb{R}^{3}, \mathfrak{k}\right)$ associated to $A$ is defined by $d_{A} \omega=d \omega+[A \wedge \omega]$, and the operator formally adjoint to $d_{A}$ with respect to scalar product (11) is equal to $-* d_{A} *$. We denote by $\operatorname{div}_{A}$ the part of this operator acting from $\Omega_{c}^{1}\left(\mathbb{R}^{3}, \mathfrak{k}\right)$ to $\Omega_{c}^{0}\left(\mathbb{R}^{3}, \mathfrak{k}\right)$, with the opposite sign,

$$
\operatorname{div}_{A}=* d_{A} *: \Omega_{c}^{1}\left(\mathbb{R}^{3}, \mathfrak{k}\right) \rightarrow \Omega_{c}^{0}\left(\mathbb{R}^{3}, \mathfrak{k}\right) .
$$

Consider the affine space of smooth connections in the trivial $K$-bundle, associated to the adjoint representation of $K$, over $\mathbb{R}^{3}$. We fix the standard trivialization of this bundle and the trivial connection as an origin in the affine space of connections and identify this space with the space $\Omega^{1}\left(\mathbb{R}^{3}, \mathfrak{k}\right)$ of $\mathfrak{k}$-valued 1 -forms on $\mathbb{R}^{3}$. We shall frequently write $\mathcal{D}$ instead of $\Omega^{1}\left(\mathbb{R}^{3}, \mathfrak{k}\right)$.

Let $\mathcal{K}$ be the group of $K$-valued smooth maps $g: \mathbb{R}^{3} \rightarrow K$. $\mathcal{K}$ is called the gauge group. The Lie algebra of $\mathcal{K}$ is isomorphic to $\Omega^{0}\left(\mathbb{R}^{3}, \mathfrak{k}\right)$.

The gauge group $\mathcal{K}$ acts on the space of connections $\mathcal{D}$ by

$$
\begin{aligned}
& \mathcal{K} \times \mathcal{D} \rightarrow \mathcal{D}, \\
& g \times A \mapsto g \circ A=-d g g^{-1}+g A g^{-1},
\end{aligned}
$$

where we denote $d g g^{-1}=g^{*} \theta_{R}, g A g^{-1}=\operatorname{Ad} g(A), \theta_{R}$ is the right-invariant MaurerCartan form on $K$, and $\operatorname{Ad} g$ stands for $g$ acting in the adjoint representation.

Action (3) induces the corresponding transformation law for the curvature $F$,

$$
g \circ F=\operatorname{Ad} g(F),
$$

where $\mathrm{Ad} g$ acts on $F$ componentwise.

For any real vector space $V$ we denote by $V_{\mathbb{C}}$ its complexification. Let $\mathcal{H}^{1}$ be the completion of the space $\Omega_{c}^{1}\left(\mathbb{R}^{3}, \mathfrak{g}\right)$ with respect to scalar product (1) extended from $\Omega_{c}^{1}\left(\mathbb{R}^{3}, \mathfrak{k}\right)$ to $\Omega_{c}^{1}\left(\mathbb{R}^{3}, \mathfrak{g}\right)$ in the natural way,

$$
<\omega_{1}, \omega_{2}>=-\int_{\mathbb{R}^{3}}\left(\omega_{1} \wedge, * \bar{\omega}_{2}\right)=-\int_{\mathbb{R}^{3}} *\left(\omega_{1} \wedge, * \bar{\omega}_{2}\right) d^{3} x, \omega_{1,2} \in \Omega^{*}\left(\mathbb{R}^{3}, \mathfrak{g}\right),
$$


where $\bar{\omega}_{2}$ is the complex conjugate of $\omega_{2}$ with respect to the complex structure induced by the decomposition $\mathfrak{g}=\mathfrak{k}+i \mathfrak{k}$. Note that $\mathfrak{k} \subset \mathfrak{g}$ is a real subspace with respect to this complex structure. We also denote by $\mathcal{H}^{0}$ the completion of the space $\Omega_{c}^{0}\left(\mathbb{R}^{3}, \mathfrak{g}\right)$ with respect to scalar product (4).

For any $A \in \mathcal{D}$ the operator $\operatorname{div}_{A}: \Omega^{1}\left(\mathbb{R}^{3}, \mathfrak{k}\right) \rightarrow \Omega^{0}\left(\mathbb{R}^{3}, \mathfrak{k}\right)$ gives rise to a linear operator $\operatorname{div}_{A}: \Omega_{c}^{1}\left(\mathbb{R}^{3}, \mathfrak{g}\right) \rightarrow \Omega_{c}^{1}\left(\mathbb{R}^{3}, \mathfrak{g}\right)$ and the closure of this operator is a welldefined closed operator $\operatorname{div}_{A}: \mathcal{H}^{1} \rightarrow \mathcal{H}^{0}$. Denote by $P_{A}: \mathcal{H}^{1} \rightarrow \operatorname{Ker} \operatorname{div}_{A}$ the orthogonal projector onto the kernel of this operator. Note that both $\operatorname{div}_{A}$ and $P_{A}$ respect the natural real structure on $\mathcal{H}^{1}$, i.e. they are real operators. Let $\left(\operatorname{Ker} \operatorname{div}_{A}\right)_{c}=\operatorname{Ker} \operatorname{div}_{A} \cap \Omega_{c}^{1}\left(\mathbb{R}^{3}, \mathfrak{g}\right)$.

For any $A \in \mathcal{D}$ we define the operator $X_{A}:\left(\operatorname{Ker} \operatorname{div}_{A}\right)_{c} \rightarrow \operatorname{Ker} \operatorname{div}_{A}$ by

$$
X_{A}=P_{A} \operatorname{curl}_{A}:\left(\operatorname{Ker} \operatorname{div}_{A}\right)_{c} \rightarrow \operatorname{Ker} \operatorname{div}_{A},
$$

where

$$
\operatorname{curl}_{A}=* d_{A}:\left(\operatorname{Ker} \operatorname{div}_{A}\right)_{c} \rightarrow \Omega_{c}^{1}\left(\mathbb{R}^{3}, \mathfrak{g}\right) .
$$

Lemma 1. The operator $X_{A}$ is real and symmetric in the sense that

$$
<X_{A} p, q>=<p, X_{A} q>, \forall p, q \in\left(\operatorname{Ker} \operatorname{div}_{A}\right)_{c},
$$

and

$$
\overline{X_{A} p}=X_{A} \bar{p}
$$

Proof.

The operator $\operatorname{curl}_{A}=* d_{A}: \Omega_{c}^{1}\left(\mathbb{R}^{3}, \mathfrak{g}\right) \rightarrow \Omega_{c}^{1}\left(\mathbb{R}^{3}, \mathfrak{g}\right)$ is a noncommutative analog of the usual curl. As in the commutative case the main property of this operator is that it is symmetric with respect to scalar product (4),

$$
<\omega_{1}, \operatorname{curl}_{A} \omega_{2}>=<\operatorname{curl}_{A} \omega_{1}, \omega_{2}>, \omega_{1,2} \in \Omega_{c}^{1}\left(\mathbb{R}^{3}, \mathfrak{g}\right) .
$$

This can be checked directly using the Stokes formula and the fact that the Killing form of $\mathfrak{g}$ is invariant with respect to the adjoint action of $\mathfrak{k}$ on $\mathfrak{g}$.

Since the operator $\operatorname{curl}_{A}$ is symmetric $X_{A}$ is also a symmetric operator. This completes the proof of the lemma.

The operators $\operatorname{curl}_{A}, X_{A}$ and $\operatorname{div}_{A}$ are gauge equivariant in the sense that

$$
\begin{gathered}
\operatorname{curl}_{g \circ A}=g \operatorname{curl}_{A} g^{-1}, \\
X_{g \circ A}=g X_{A} g^{-1}, \\
\operatorname{div}_{g \circ A}=g \operatorname{div}_{A} g^{-1} .
\end{gathered}
$$

In these formulas it is assumed that the action of the gauge group $\mathcal{K}$ on the space $\Omega_{c}^{*}\left(\mathbb{R}^{3}, \mathfrak{g}\right)$ is induced by the adjoint representation, $g \omega=\operatorname{Ad} g(\omega), g \in \mathcal{K}, \omega \in$ $\Omega_{c}^{*}\left(\mathbb{R}^{3}, \mathfrak{g}\right)$. Since the Killing form is invariant under the adjoint action the gauge group $\mathcal{K}$ also acts on the spaces $\mathcal{H}^{0}$ and $\mathcal{H}^{1}$ by unitary transformations.

We shall prove that the operators $X_{A}:\left(\operatorname{Ker} \operatorname{div}_{A}\right)_{c} \rightarrow \operatorname{Ker} \operatorname{div}_{A}$ have selfadjoint extensions $X_{A}$ : Ker $\operatorname{div}_{A} \rightarrow \operatorname{Ker} \operatorname{div}_{A}$ for $A$ close enough to 0 with respect to a certain topology on $\mathcal{D}$, and those selfadjoint extensions are unitary equivalent to each other. We start realizing this program by recalling scattering theory for selfadjoint operators in the form suitable for our purposes. 


\section{WAVE OPERATORS AND UNITARY EQUIVALENCE}

Let $\mathfrak{H}$ be a complex Hilbert space. In this section we recall, following [7, some results on unitary equivalence for selfadjoint operators on $\mathfrak{H}$. Let $\mathcal{C}_{0}(\mathfrak{H})$ be the set of closed densely defined linear operators $T$ on $\mathfrak{H}$ with domain $\mathfrak{D}(T)$ and range $\Re(T), \mathcal{B}(\mathfrak{H})$ the set of bounded operators on $\mathfrak{H}$.

Let $T_{0}$ be a selfadjoint operator in $\mathfrak{H}$ and $U \in \mathcal{C}_{0}(\mathfrak{H})$. Denote by $R_{0}(\zeta)=$ $\left(T_{0}-\zeta\right)^{-1}$ the resolvent of $T_{0}$. As a function of $\zeta$ the resolvent $R_{0}(\zeta)$ is holomorphic in the open lower and upper half-plane.

Suppose that $\mathfrak{D}(U) \supset \mathfrak{D}\left(T_{0}\right)$. Then $U R_{0}(\zeta) \in \mathcal{B}(\mathfrak{H})$ for $\operatorname{Im} \zeta \neq 0$, and $U R_{0}(\zeta)$ is holomorphic in the open lower and upper half-plane. The operator $U$ is said to be $T_{0}$-smooth if for each $u \in \mathfrak{H}$ there is a constant $M_{u}$ independent of $\varepsilon$ such that

$$
\int_{-\infty}^{\infty}\left\|U R_{0}(\lambda \pm i \varepsilon) u\right\|^{2} d \lambda \leq M_{u}^{2}, \forall \varepsilon>0 .
$$

The following proposition gives a strong version of the well-known construction for the wave operators.

Proposition 2. (13, Theorems XIII.24, XIII.26) Let $T_{0}, T$ be two selfadjoint operators in Hilbert space $\mathfrak{H}$ such that $T=T_{0}+\sum_{i=1}^{n} V_{i}^{*} U_{i}$, where $U_{i}, V_{i} \in \mathcal{C}_{0}(\mathfrak{H})$ and $U_{i}, V_{i}$ are $T_{0}$-smooth. Denote by $R_{0}(\zeta)$ the resolvent of $T_{0}$ and assume that

$$
A_{i j}=\sup _{\zeta \notin \mathbb{R}}\left\|U_{i} R_{0}(\zeta) V_{j}^{*}\right\|<\infty, i, j=1, \ldots, n .
$$

Assume, furthermore, that the norm of the matrix $A=\left\{A_{i j}\right\}_{i, j=1, \ldots, n}$, regarded as an operator in $\mathbb{C}^{n}$ with the natural Hilbert space norm, is strictly less than 1 ,

$$
\|A\|<1 \text {. }
$$

Then the wave operators

$$
W_{ \pm}=s-\lim _{t \rightarrow \pm \infty} e^{i T t} e^{-i T_{0} t}
$$

for the pair $T_{0}, T$ exist and are unitary operators, i.e. $W_{ \pm}^{-1} \in \mathcal{B}(\mathfrak{H})$. In particular, the operators $T_{0}$ and $T$ are unitary equivalent,

$$
T=W_{ \pm} T_{0} W_{ \pm}^{-1}
$$

The condition of $T_{0}$-smoothness is usually difficult to verify. The following proposition gives a simple sufficient criterion of $T_{0}$-smoothness.

Proposition 3. ([13, Corollary of Theorem XIII.25) Let $T_{0}$ be a selfadjoint operator in $\mathfrak{H}, U \in \mathcal{C}_{0}(\mathfrak{H})$. Then $U$ is $T_{0}$-smooth if

$$
\sup \left\|U R_{0}(\zeta) U^{*}\right\|<\infty,
$$

where the supremum is taken over all $\zeta \in \mathbb{C}$ with $\operatorname{Im} \zeta \neq 0$.

\section{SCATtering THEORY FOR the operators $X_{A}$ : EXtension to Elliptic OPERATORS}

The main obstruction to direct application of Proposition 2 to any selfadjoint extensions of the operators $X_{A}$ is that for different $A$ these operators act in different spaces, $X_{A}:\left(\operatorname{Ker} \operatorname{div}_{A}\right)_{c} \rightarrow \operatorname{Ker} \operatorname{div}_{A}$. However, using formula (5) one can naturally extend these operators to symmetric operators acting in $\mathcal{H}^{2}$ with the domains 
$\left(\text { Ker } \operatorname{div}_{A}\right)_{c} \oplus\left(\operatorname{Ker} \operatorname{div}_{A}\right)_{c}^{\perp}$, where $\left(\operatorname{Ker} \operatorname{div}_{A}\right)_{c}^{\perp}=\left(\operatorname{Ker} \operatorname{div}_{A}\right)^{\perp} \cap \Omega_{c}^{1}\left(\mathbb{R}^{3}, \mathfrak{g}\right)$. If we denote these extensions also by $X_{A}$ then

$$
X_{A}=P_{A} \operatorname{curl}_{A} P_{A},
$$

i.e., we extend $X_{A}$ : $\left(\operatorname{Ker} \operatorname{div}_{A}\right)_{c} \rightarrow\left(\operatorname{Ker} \operatorname{div}_{A}\right)$ by zero to the orthogonal complement $\left(\operatorname{Ker} \operatorname{div}_{A}\right)_{c}$ of its original domain. Therefore the operators defined by formula (10) have big kernels.

As Remark 1.9 in [7] shows, in order to establish unitary equivalence for operators with nonempty point spectrum we have to significantly restrict the class of perturbations (actually if $u$ is an eigenvector of $T_{0}$, in the notation of Proposition 2. then for $U$ to be $T_{0}$-smooth we must have $U u=0$ ). This is not satisfactory for our purposes.

In order to overcome this difficulty we shall find an elliptic extension $S_{A}$ for the operator $X_{A}$ (compare with [2, 3]). $S_{A}$ is a symmetric operator acting in the space $\mathcal{H}^{1}+\mathcal{H}^{0}$ with the domain $\mathfrak{D}\left(S_{A}\right)=\left(\left(\operatorname{Ker} \operatorname{div}_{A}\right)_{c} \oplus\left(\operatorname{Ker} \operatorname{div}_{A}\right)_{c}^{\perp}\right)+\Omega_{c}^{0}\left(\mathbb{R}^{3}, \mathfrak{g}\right)$. If we represent $S_{A}$ in the block form then

$$
S_{A}=\left(\begin{array}{cc}
P_{A} \operatorname{curl}_{A} P_{A} & -d_{A} \\
\operatorname{div}_{A} & 0
\end{array}\right) .
$$

In Section [6 we shall define natural selfadjoint extensions for $S_{A}$ and obtain wave operators for selfadjoint extensions of operators $X_{A}$ using those for selfadjoint extensions of $S_{A}$.

\section{The Properties of the unPerturbed operator $S_{0}$}

In order to apply Proposition 2 to the operators $S_{A}$ we have to study first the unperturbed operator $S_{0}$. In particular, in view of condition (7) we have to study the properties of the resolvent of the operator $S_{0}$.

First we note that the operator $S_{0}$,

$$
S_{0}=\left(\begin{array}{cc}
\operatorname{curl} & -d \\
\operatorname{div} & 0
\end{array}\right)
$$

acting in the space $\mathcal{H}^{1} \dot{+} \mathcal{H}^{0}$ with the natural domain

$$
\mathfrak{D}\left(S_{0}\right)=\left\{(\omega, u) \in \mathcal{H}^{1} \dot{+} \mathcal{H}^{0}: \operatorname{curl} \omega, d u \in \mathcal{H}^{1}, \operatorname{div} \omega \in \mathcal{H}^{0}\right\}
$$

is selfadjoint (see 2] 3]). The formula for the resolvent of this operator can easily be obtained with the help of an explicit expression for $S_{0}^{2}$,

$$
S_{0}^{2}=\left(\begin{array}{cc}
\widehat{\triangle} & 0 \\
0 & \triangle
\end{array}\right)
$$

where $\triangle$ is the usual Laplace operator and $\widehat{\triangle}$ stands for the Laplace operator acting on the components of elements from $\mathcal{H}^{1}$. If we denote the resolvent of $S_{0}$ by $R_{0}(\lambda)$ then (see 4] for a similar calculation for the operator curl)

$$
R_{0}(\lambda)=\left(S_{0}-\lambda I\right)^{-1}=\left(S_{0}+\lambda I\right)\left(S_{0}^{2}-\lambda^{2} I\right)^{-1},
$$


or in components

$$
R_{0}(\lambda)=\left(\begin{array}{cc}
\operatorname{curl}+\lambda I & -d \\
\operatorname{div} & \lambda I
\end{array}\right)\left(\begin{array}{cc}
\left(\widehat{\triangle}-\lambda^{2} I\right)^{-1} & 0 \\
0 & \left(\triangle-\lambda^{2} I\right)^{-1}
\end{array}\right)=
$$

$$
\left(\begin{array}{cc}
(\operatorname{curl}+\lambda I)\left(\widehat{\triangle}-\lambda^{2} I\right)^{-1} & -d\left(\triangle-\lambda^{2} I\right)^{-1} \\
\operatorname{div}\left(\widehat{\triangle}-\lambda^{2} I\right)^{-1} & \lambda\left(\triangle-\lambda^{2} I\right)^{-1}
\end{array}\right) .
$$

Verification of condition (77) will be based on the fact that the resolvent $R_{0}(\lambda)$ acts as a bounded operator in certain weighted $L^{2}$-spaces.

We recall that for any real $s$ the weighted space $L^{2, s}\left(\mathbb{R}^{n}\right)$ is defined by

$$
L^{2, s}\left(\mathbb{R}^{n}\right)=\left\{u(x):\left(1+|x|^{2}\right)^{\frac{s}{2}} u(x) \in L^{2}\left(\mathbb{R}^{n}\right)\right\},
$$

where $|x|$ is the usual norm of $x$ in $\mathbb{R}^{n}$. The space $L^{2, s}\left(\mathbb{R}^{n}\right)$ is a Hilbert space, the corresponding norm $\|\cdot\|_{s}$ is equal to

$$
\|u\|_{s}=\left\|\left(1+|x|^{2}\right)^{\frac{s}{2}} u(x)\right\|_{L^{2}\left(\mathbb{R}^{n}\right)} .
$$

Clearly, the spaces $L^{2, s}\left(\mathbb{R}^{n}\right)$ and $L^{2,-s}\left(\mathbb{R}^{n}\right)$ are dual to each other.

We shall denote by $\mathcal{H}^{0, s}$ and $\mathcal{H}^{1, s}$ the weighted versions of the spaces $\mathcal{H}^{0}$ and $\mathcal{H}^{1}$, with the obvious modifications of the scalar products.

For any bounded operator $T: L^{2, s_{1}}\left(\mathbb{R}^{n}\right) \rightarrow L^{2, s_{2}}\left(\mathbb{R}^{n}\right)$ we denote by $\|T\|_{s_{1}, s_{2}}$ its norm. The required estimates for the resolvent $R_{0}$ will be based on the following well-known results by Agmon and Jensen-Kato on the resolvent of the Laplace operator.

Proposition 4. ([1, Appendix A, Remark 2; [6, Lemma 2.1) Let $(\triangle-\zeta I)^{-1}$ be the resolvent of the Laplace operator acting in $L^{2}\left(\mathbb{R}^{3}\right)$. Then for any $\lambda \in \mathbb{C}$ and $s>1$ the operators

$$
\lambda\left(\triangle-\lambda^{2} I\right)^{-1}, \partial_{i}\left(\triangle-\lambda^{2} I\right)^{-1}, i=1,2,3,
$$

act as bounded operators from $L^{2, s}\left(\mathbb{R}^{n}\right)$ to $L^{2,-s}\left(\mathbb{R}^{n}\right)$. Moreover,

$$
\begin{aligned}
& \left\|\lambda\left(\triangle-\lambda^{2} I\right)^{-1}\right\|_{s,-s} \leq C, \\
& \left\|\partial_{i}\left(\triangle-\lambda^{2} I\right)^{-1}\right\|_{s,-s} \leq C, \quad i=1,2,3,
\end{aligned}
$$

where $C$ is a constant independent of $\lambda$.

Fix $\varepsilon>0$. Then for any $\lambda \in \mathbb{C}$ with $|\lambda| \geq \varepsilon$ and $s>\frac{1}{2}$ the operators

$$
\frac{1}{\lambda} \partial_{i} \partial_{j}\left(\triangle-\lambda^{2} I\right)^{-1}, i, j=1,2,3
$$

act as bounded operators from $L^{2, s}\left(\mathbb{R}^{n}\right)$ to $L^{2,-s}\left(\mathbb{R}^{n}\right)$. Moreover,

$$
\left\|\frac{1}{\lambda} \partial_{i} \partial_{j}\left(\triangle-\lambda^{2} I\right)^{-1}\right\|_{s,-s} \leq C, i=1,2,3
$$

where $C$ is a constant independent of $\lambda$.

Clearly, the blocks of the resolvent $R_{0}(\lambda)$ consist of the terms of form (15) (see formula (14)). Therefore Proposition 4 provides an estimate uniform in $\lambda$ of the norm of the resolvent $R_{0}(\lambda)$ acting as a bounded operator from $\mathcal{H}^{1, s}+\mathcal{H}^{0, s}$ to $\mathcal{H}^{1,-s}+\mathcal{H}^{0,-s}$. We formulate this result as a corollary to Proposition 4 
Corollary 5. For any $\lambda \in \mathbb{C}$ and $s>1$ the resolvent $R_{0}(\lambda)$ acts as a bounded operator from $\mathcal{H}^{1, s}+\mathcal{H}^{0, s}$ to $\mathcal{H}^{1,-s}+\mathcal{H}^{0,-s}$. Moreover,

$$
\left\|R_{0}(\lambda)\right\|_{s,-s} \leq C,
$$

where $C$ is a constant independent of $\lambda$.

\section{SCATtering theory FOR the Elliptic OPERATORS $S_{A}$}

In this section we find conditions under which the operators $S_{0}$ and $S_{A}$ are unitary equivalent. In order to derive these conditions we apply Proposition 2 to the pair $S_{0}, S_{A}$. First we formulate our main result.

Theorem 6. Let $A$ be a $\mathfrak{k}$-valued connection 1-form on $\mathbb{R}^{3}, A \in \Omega^{1}\left(\mathbb{R}^{3}, \mathfrak{k}\right), F$ the curvature of $A$. There exists a positive constant $C$ such that if for some $s>3$ and $g \in \mathcal{K}$

$$
\begin{aligned}
& \left\|g \circ F\left(1+|x|^{2}\right)^{s}\right\|_{(\infty)}<C, \\
& \left\|g \circ A\left(1+|x|^{2}\right)^{\frac{s}{2}}\right\|_{(\infty)}<C,
\end{aligned}
$$

where for any $\omega \in \Omega^{i}\left(\mathbb{R}^{3}, \mathfrak{g}\right)$

$$
\|\omega\|_{(\infty)}=\operatorname{ess}-\sup _{x \in \mathbb{R}^{3}} *(\omega(x) \wedge, * \overline{\omega(x)})^{\frac{1}{2}},
$$

then

(i) The corresponding operator $S_{A}$ is selfadjoint with the domain $g^{-1} \mathfrak{D}\left(S_{0}\right)$.

(ii) The wave operators

$$
W_{ \pm}\left(S_{0}, S_{A}\right)=s-\lim _{t \rightarrow \pm \infty} e^{i S_{A} t} e^{-i S_{0} t}
$$

for the pair $S_{0}, S_{A}$ exist and are unitary. In particular, the selfadjoint operators $S_{0}$ and $S_{A}$ are unitary equivalent.

Remark 7. Since the operators $S_{A}$ are gauge equivariant and the gauge group $\mathcal{K}$ acts on the space $\mathcal{H}^{1}$ by unitary transformations we shall assume, without loss of generality, that conditions (16), (17) are imposed on $F$ and $A$, or, in other words, that $A$ is in the gauge in which the conditions (16), (17) are satisfied.

We start the proof of this theorem with the study of the orthogonal projection operator $P_{A}$ and the "magnetic" Laplace operator $\triangle_{A}=-\operatorname{div}_{A} d_{A}$.

Lemma 8. Let $\mathcal{H}_{\text {loc }}^{1}\left(\mathbb{R}^{3}, \mathfrak{k}\right)$ be the space of locally square integrable, with respect to the scalar product (1), $\mathfrak{k}$-valued 1 -forms on $\mathbb{R}^{3}$. Then for any $A \in \mathcal{H}_{\text {loc }}^{1}\left(\mathbb{R}^{3}, \mathfrak{k}\right)$ we have:

(i) The operators $-d_{A}: \Omega_{c}^{0}\left(\mathbb{R}^{3}, \mathfrak{g}\right) \rightarrow \mathcal{H}^{1}$ and $\operatorname{div}_{A}: \Omega_{c}^{1}\left(\mathbb{R}^{3}, \mathfrak{g}\right) \rightarrow \mathcal{H}^{0}$ are closable and their closures are operators $-d_{A}: \mathcal{H}^{0} \rightarrow \mathcal{H}^{1}, \operatorname{div}_{A}: \mathcal{H}^{1} \rightarrow \mathcal{H}^{0}$ which are adjoint to each other.

(ii) The operator $\triangle_{A}: \mathcal{H}^{0} \rightarrow \mathcal{H}^{0}$ defined by the differential expression $\triangle_{A}=$ $-\operatorname{div}_{A} d_{A}$ is selfadjoint on the natural domain $\mathfrak{D}\left(\triangle_{A}\right)=\left\{u \in \mathfrak{D}\left(d_{A}\right) \mid d_{A} u \in\right.$ $\left.\mathfrak{D}\left(\operatorname{div}_{A}\right)\right\}$.

(iii) The operator $\triangle_{A}$ has trivial kernel, and the inverse operator $\triangle_{A}^{-1}$ is welldefined.

(iv) The operator $P_{A}$ is the closure of the operator $I+d_{A} \triangle_{A}^{-1} \operatorname{div}_{A}$ defined on $\Re\left(d_{A}\right) \oplus \operatorname{Ker} \operatorname{div}_{A}$. 
Proof. For parts (i) and (ii) see [11, §X.3, Example 4.

In order to prove part (iii) we observe that if $u \in \operatorname{Ker} \triangle_{A}$ then $d_{A} u=0$ since $0=\left(\triangle_{A} u, u\right)=-\left(\operatorname{div}_{A} d_{A} u, u\right)=\left(d_{A} u, d_{A} u\right)$. But then by the invariance of the Killing form we have

$$
d(u, u)=\left(d_{A} u, u\right)+\left(u, d_{A} u\right)=0 .
$$

Therefore $(u, u)=$ const, and $u$ does not belong to $\mathcal{H}^{0}$.

For part (iv) we first note that the orthogonal direct sum $\Re\left(d_{A}\right) \bigoplus \operatorname{Ker} \operatorname{div}_{A}$ is dense in $\mathcal{H}^{1}, \mathcal{H}^{1}=\overline{\Re\left(d_{A}\right)} \bigoplus$ Ker $\operatorname{div}_{A}$

Next, if $\omega \in \Re\left(d_{A}\right), \omega=d_{A} u$ then $\left(I+d_{A} \triangle_{A}^{-1} \operatorname{div}_{A}\right) \omega=\omega+d_{A} \triangle_{A}^{-1} \operatorname{div}_{A} d_{A} u=0$ since $\triangle_{A}^{-1} \operatorname{div}_{A} d_{A} u=-u$, and if $\omega \in \operatorname{Ker} \operatorname{div}_{A}$ then $\left(I+d_{A} \triangle_{A}^{-1} \operatorname{div}_{A}\right) \omega=\omega$. This completes the proof.

Now we write down the perturbation $W=S_{A}-S_{0}$ in a convenient form. From formula (11) we formally have

$$
W=\left(\begin{array}{cc}
P_{0} * \operatorname{ad} A P_{0}-P_{0} \operatorname{curl}_{A} \Delta P-\Delta P \operatorname{curl}_{A} P_{0}+\Delta P \operatorname{curl}_{A} \Delta P & -\operatorname{ad} A \\
* \operatorname{ad} A * & 0
\end{array}\right),
$$

where $\operatorname{ad} A(\omega)=[A \wedge \omega], \omega \in \Omega^{i}\left(\mathbb{R}^{3}, \mathfrak{g}\right)$, and $\Delta P=P_{0}-P_{A}$.

Using the expression for the operator $P_{A}$ obtained in part (iv) of Lemma 8 the Hilbert-type identity,

$$
\triangle_{A}^{-1}=\triangle^{-1}-\triangle^{-1}\left(\triangle_{A}-\triangle\right) \triangle_{A}^{-1},
$$

and the formula

$$
\triangle_{A}-\triangle=-\operatorname{div} \operatorname{ad} A-* \operatorname{ad} A * d-* \operatorname{ad} A * \operatorname{ad} A
$$

one can derive the following formal expression for $\Delta P$ :

$$
\begin{array}{rl}
\Delta P=d \triangle^{-1} & * \operatorname{ad} A *+\operatorname{ad} A \triangle^{-1} \operatorname{div}_{A}+ \\
& +d_{A} \triangle^{-1} \operatorname{div} \operatorname{ad} A \triangle_{A}^{-1} \operatorname{div}_{A}+d_{A} \triangle^{-1} * \operatorname{ad} A * d_{A} \triangle_{A}^{-1} \operatorname{div}_{A} .
\end{array}
$$

Substituting (19) into formula (18) we get useful expressions for the terms of the perturbation $W$,

$$
\begin{aligned}
& \triangle P \operatorname{curl}_{A} P_{0}=\left(d \triangle^{-1} * \operatorname{ad} F-d \triangle^{-1} \operatorname{div} * \operatorname{ad} A+\operatorname{ad} A \triangle^{-1} * \operatorname{ad} F+\right. \\
&+d \triangle^{-1} \operatorname{div} \operatorname{ad} A \triangle_{A}^{-1} * \operatorname{ad} F+d \triangle^{-1} * \operatorname{ad} A * d_{A} \triangle_{A}^{-1} * \operatorname{ad} F+ \\
&\left.+\operatorname{ad} A \triangle^{-1} \operatorname{div} \operatorname{ad} A \triangle_{A}^{-1} * \operatorname{ad} F+\operatorname{ad} A \triangle^{-1} * \operatorname{ad} A * d_{A} \triangle_{A}^{-1} * \operatorname{ad} F\right) P_{0}, \\
& P_{0} \operatorname{curl}_{A} \Delta P=P_{0}\left(* \operatorname{ad} F \triangle^{-1} \operatorname{div}-* \operatorname{ad} A d \triangle^{-1} \operatorname{div}+* \operatorname{ad} F \triangle^{-1} * \operatorname{ad} A *+\right. \\
&+ * \operatorname{ad} F \triangle_{A}^{-1} * \operatorname{ad} A * d \triangle^{-1} \operatorname{div}+* \operatorname{ad} F \triangle_{A}^{-1} \operatorname{div}_{A} \operatorname{ad} A \triangle^{-1} \operatorname{div}+ \\
&\left.+* \operatorname{ad} F \triangle_{A}^{-1} * \operatorname{ad} A * d \triangle^{-1} * \operatorname{ad} A *+* \operatorname{ad} F \triangle_{A}^{-1} \operatorname{div}_{A} \operatorname{ad} A \triangle^{-1} * \operatorname{ad} A *\right),
\end{aligned}
$$

$\Delta P \operatorname{curl}_{A} \Delta P=\left(d \triangle^{-1} * \operatorname{ad} A *+\operatorname{ad} A \triangle^{-1} \operatorname{div}+\operatorname{ad} A \triangle^{-1} * \operatorname{ad} A *+\right.$

$$
+d \triangle^{-1} \operatorname{div} \operatorname{ad} A \triangle_{A}^{-1} \operatorname{div}_{A}+\operatorname{ad} A \triangle^{-1} \operatorname{div} \operatorname{ad} A \triangle_{A}^{-1} \operatorname{div}_{A}+
$$

$$
\left.+d \triangle^{-1} * \operatorname{ad} A * d_{A} \triangle_{A}^{-1} \operatorname{div}_{A}+\operatorname{ad} A \triangle^{-1} * \operatorname{ad} A * d_{A} \triangle_{A}^{-1} \operatorname{div}_{A}\right) \times
$$

$\times\left(* \operatorname{ad} F \triangle^{-1} \operatorname{div}-* \operatorname{ad} A d \triangle^{-1} \operatorname{div}+* \operatorname{ad} F \triangle^{-1} * \operatorname{ad} A *+\right.$

$$
+* \operatorname{ad} F \triangle_{A}^{-1} * \operatorname{ad} A * d \triangle^{-1} \operatorname{div}+* \operatorname{ad} F \triangle_{A}^{-1} \operatorname{div}{ }_{A} \operatorname{ad} A \triangle^{-1} \operatorname{div}+
$$

$$
\left.+* \operatorname{ad} F \triangle_{A}^{-1} * \operatorname{ad} A * d \triangle^{-1} * \operatorname{ad} A *+* \operatorname{ad} F \triangle_{A}^{-1} \operatorname{div}_{A} \operatorname{ad} A \triangle^{-1} * \operatorname{ad} A *\right) \text {. }
$$


In order to analyze expressions (20)-(22) we shall need the properties of $\triangle_{A}^{-1}$ as an operator acting in weighted $L^{2}$-spaces and $L^{p}$-spaces. For any $p \geq 1$ denote by $\mathcal{H}_{p}^{i}$ the closure of the space $\Omega_{c}^{i}\left(\mathbb{R}^{3}, \mathfrak{g}\right)$ with respect to the norm

$$
\begin{gathered}
\|\omega\|_{(p)}=\left(-\int_{\mathbb{R}^{3}} *(\omega \wedge, * \bar{\omega})^{\frac{p}{2}} d^{3} x\right)^{\frac{1}{p}}, p<\infty, \\
\|\omega\|_{(\infty)}=\text { ess }-\sup _{x \in \mathbb{R}^{3}} *(\omega(x) \wedge, * \overline{\omega(x)})^{\frac{1}{2}} .
\end{gathered}
$$

Note that from the Hölder inequality it follows that for any $p \geq 2$ we have a natural embedding, $\mathcal{H}_{p}^{i} \subset \mathcal{H}^{i,-s}$, where $s>3\left(\frac{1}{2}-\frac{1}{p}\right)$, and for any $1 \leq p \leq 2$ we have another embedding, $\mathcal{H}^{i, s} \subset \mathcal{H}_{p}^{i}$, where $s>3\left(\frac{2}{p}-1\right)$, i.e.,

$$
\begin{aligned}
& \mathcal{H}_{p}^{i} \subset \mathcal{H}^{i,-s}, p \geq 2, s>3\left(\frac{1}{2}-\frac{1}{p}\right), \\
& \mathcal{H}^{i, s} \subset \mathcal{H}_{p}^{i}, 1 \leq p \leq 2, s>3\left(\frac{2}{p}-1\right) .
\end{aligned}
$$

Obviously, there are also natural embeddings

$$
\mathcal{H}^{i, s_{1}} \subset \mathcal{H}^{i, s_{2}} \subset \mathcal{H}^{i} \subset \mathcal{H}^{i,-s_{2}} \subset \mathcal{H}^{i,-s_{1}}, s_{1}>s_{2}>0 .
$$

Lemma 9. (i) For any $A \in \mathcal{H}_{\text {loc }}^{1}\left(\mathbb{R}^{3}, \mathfrak{k}\right), u \in \mathcal{H}_{\frac{6}{5}}^{0}$ and $\omega \in \mathcal{H}_{2}^{1}$ we have

$$
\begin{array}{r}
\left\|\triangle_{A}^{-1} u\right\|_{(6)} \leq K^{2}\|u\|_{\left(\frac{6}{5}\right)}, \\
\left\|d_{A} \triangle_{A}^{-1} u\right\|_{(2)} \leq K\|u\|_{\left(\frac{6}{5}\right)}, \\
\left\|\triangle_{A}^{-1} \operatorname{div}_{A} \omega\right\|_{(6)} \leq K\|\omega\|_{(2)},
\end{array}
$$

where $K$ is a constant independent of $A$.

(ii) The following operators are bounded and have norms independent of A:

$$
\begin{aligned}
& \triangle_{A}^{-1}: \mathcal{H}^{0, s_{1}} \rightarrow \mathcal{H}^{0,-s_{2}}, s_{1}>2, s_{2}>1, \\
& \triangle_{A}^{-1}: \mathcal{H}^{0, s_{1}} \rightarrow \mathcal{H}^{0,-s_{2}}, s_{1}>1, s_{2}>2 \\
& \triangle_{A}^{-1} \operatorname{div}_{A}: \mathcal{H}^{1} \rightarrow \mathcal{H}^{0,-s}, s>1 \\
& d_{A} \triangle_{A}^{-1}: \mathcal{H}^{0, s} \rightarrow \mathcal{H}^{1}, s>1 .
\end{aligned}
$$

Proof. Inequality (25) follows from the well-known Kato inequality, $\|d \mid u\|_{(2)} \leq$ $\left\|d_{A} u\right\|_{(2)}$ (see [5], Appendix), and the isoperimetric-type inequality, $\|f\|_{(6)} \leq K\|d f\|_{(2)}$, $f \in C_{0}^{\infty}\left(\mathbb{R}^{3}\right)$ (see $[\underline{8}$, Theorem 2.1). Indeed, these two inequalities and the Hölder inequality imply that

$$
\|w\|_{(6)}^{2} \leq K^{2}\|d|w|\|_{(2)}^{2} \leq K^{2}\left(\triangle_{A} w, w\right) \leq K^{2}\|w\|_{(6)}\left\|\triangle_{A} w\right\|_{\left(\frac{6}{5}\right)} .
$$

This inequality holds for $w \in \mathcal{C}$, where $\mathcal{C}=\left\{\varphi v \mid \varphi \in C_{0}^{\infty}\left(\mathbb{R}^{3}\right), v \in\left(\triangle_{A}+\right.\right.$ $\left.1)^{-1}\left(\mathcal{H}_{2}^{0} \bigcap \mathcal{H}_{\infty}^{0}\right)\right\}$ is an operator core of $\triangle_{A}$ (see 9 , Lemma 5).

Now dividing both sides of (29) by $\|w\|_{(6)}$ and denoting $u=\triangle_{A} w$ we get (25).

Similarly, for any $u=\triangle_{A} w, w \in \mathcal{C}$ inequality (25) and the Hölder inequality yield

$$
\left\|d_{A} \triangle_{A}^{-1} u\right\|_{(2)}^{2}=\left(\triangle_{A} \triangle_{A}^{-1} u, \triangle_{A}^{-1} u\right)=\left(u, \triangle_{A}^{-1} u\right) \leq\left\|\triangle_{A}^{-1} u\right\|_{(6)}\|u\|_{\left(\frac{6}{5}\right)} \leq K^{2}\|u\|_{\left(\frac{6}{5}\right)}^{2} \text {, }
$$

which is equivalent to (26).

Now we deduce (27) from (26) by duality. 
Finally we infer part (ii) of the lemma using part (i), embeddings (23) and duality $\left(\mathcal{H}^{i, s}\right)^{*} \simeq \mathcal{H}^{i,-s}$.

Proof of part (i) of Theorem [6. First we note that conditions (16) and (17) ensure that for any real $s^{\prime}$

$$
\begin{aligned}
& \operatorname{ad} F: \mathcal{H}^{i, s^{\prime}} \rightarrow \mathcal{H}^{i+2, s^{\prime}+2 s}, \\
& \operatorname{ad} A: \mathcal{H}^{i, s^{\prime}} \rightarrow \mathcal{H}^{i+1, s^{\prime}+s}
\end{aligned}
$$

are bounded operators with norms not exceeding $C$. Combining this fact with part (ii) of Lemma 9 and recalling embeddings (24) we infer that the operators $* \operatorname{ad} A: \mathcal{H}^{1} \rightarrow \mathcal{H}^{1}, \operatorname{ad} A: \mathcal{H}^{0} \rightarrow \mathcal{H}^{1}, * \operatorname{ad} A *: \mathcal{H}^{1} \rightarrow \mathcal{H}^{0}$ are bounded and the operators defined by formulas (20)-(22) are bounded in the space $\mathcal{H}^{1}$. Therefore the perturbation $W$ is a bounded operator, and part (i) of Theorem 6 follows from the Kato-Rellich theorem (see [11, Theorem X.12).

Now we discuss part (ii) of Theorem [6. In order to apply Theorem 2 to the pair of operators $S_{0}, S_{A}$ we have to factorize every term of the perturbation $W$ and then verify conditions (8), (9). Note that the perturbation $W$ contains not only multiplication operators but also integral operators (see formulas (20)-(22) and to check conditions (8), (9) we shall need not only estimates for the norm of the resolvent $R_{0}(\lambda)$ of the unperturbed operator $S_{0}$ obtained in Proposition 4 and Corollary [5] but also estimates of norms of compositions of $R_{0}(\lambda)$ and of some integral operators. More precisely, in view of (20)-22), we have to obtain the following estimates.

Lemma 10. For every $\lambda \in \mathbb{C}$ the operators

$$
\begin{gathered}
-\triangle^{-1} \operatorname{div}(\operatorname{curl}+\lambda I)\left(\widehat{\triangle}-\lambda^{2} I\right)^{-1} d \triangle^{-1}= \\
=\lambda \triangle^{-1}\left(\triangle-\lambda^{2} I\right)^{-1}: \mathcal{H}^{0, s_{1}} \rightarrow \mathcal{H}^{0,-s_{2}}, s_{1}>3, s_{2}>\frac{3}{2} \\
(\operatorname{curl}+\lambda I)\left(\widehat{\triangle}-\lambda^{2} I\right)^{-1} d \triangle^{-1}= \\
=\lambda d \triangle^{-1}\left(\triangle-\lambda^{2} I\right)^{-1}: \mathcal{H}^{0, s} \rightarrow \mathcal{H}^{1,-s}, s>1 \\
\triangle^{-1} \operatorname{div}(\operatorname{curl}+\lambda I)\left(\widehat{\triangle}-\lambda^{2} I\right)^{-1}= \\
=\lambda \operatorname{div} \widehat{\triangle}^{-1}\left(\widehat{\triangle}-\lambda^{2} I\right)^{-1}: \mathcal{H}^{1, s} \rightarrow \mathcal{H}^{0,-s}, s>1 \\
(\operatorname{curl}+\lambda I)\left(\widehat{\triangle}-\lambda^{2} I\right)^{-1} d \triangle^{-1} \operatorname{div}= \\
=\lambda\left(\widehat{\triangle}-\lambda^{2} I\right)^{-1} d \triangle^{-1} \operatorname{div}: \mathcal{H}^{1, s} \rightarrow \mathcal{H}^{1,-s}, s>1 \\
-d \triangle^{-1} \operatorname{div}(\operatorname{curl}+\lambda I)\left(\triangle^{-}-\lambda^{2} I\right)^{-1} d \triangle^{-1} \operatorname{div}= \\
=\lambda\left(\widehat{\triangle}-\lambda^{2} I\right)^{-1} d \triangle^{-1} \operatorname{div}: \mathcal{H}^{1, s} \rightarrow \mathcal{H}^{1,-s}, s>1
\end{gathered}
$$

are bounded and have norms not exceeding $D$, where $D$ is a constant independent of $\lambda$.

Proof. First we recall that the resolvent of the Laplace operator is an integral operator explicitly given by the following formula (see [1])

$$
\left(\left(\triangle-\lambda^{2} I\right)^{-1} u\right)(x)=\int_{\mathbb{R}^{3}} \frac{e^{i \lambda \operatorname{sgn}(\operatorname{Im} \lambda)|x-y|}}{4 \pi|x-y|} u(y) d^{3} y
$$


To prove (31) we shall use the Hilbert identity in the form

$$
\lambda \triangle^{-1}\left(\triangle-\lambda^{2} I\right)^{-1}=\frac{1}{\lambda}\left(\left(\triangle-\lambda^{2} I\right)^{-1}-\triangle^{-1}\right) .
$$

From Proposition 4 it follows that for any $\varepsilon>0$ and $|\lambda|>\varepsilon$ the operator in the r.h.s. of (37) is a bounded operator acting from $\mathcal{H}^{0, s_{1}}$ to $\mathcal{H}^{0,-s_{2}}$ for any $s_{1,2}>1$, with the norm uniformly bounded in $\lambda$. We have to check that the norm of this operator remains finite when $\lambda \rightarrow 0$ and $s_{1}>3, s_{2}>\frac{3}{2}$.

Indeed, using formulas (36) and (37) we obtain that for any $u \in \Omega_{c}^{0}\left(\mathbb{R}^{3}, \mathfrak{g}\right)$

$$
\lim _{\lambda \rightarrow 0} \lambda \triangle^{-1}\left(\triangle-\lambda^{2} I\right)^{-1} u=\frac{i}{4 \pi} \int_{\mathbb{R}^{3}} u(y) d^{3} y .
$$

The operator in the r.h.s. of the last formula is bounded from $\mathcal{H}_{1}^{0}$ to $\mathcal{H}_{\infty}^{0}$, and hence, in view of embeddings (23), from $\mathcal{H}^{0, s_{1}}$ to $\mathcal{H}^{0,-s_{2}}$ for $s_{1}>3, s_{2}>\frac{3}{2}$. This proves (31).

(32) and (33) can be proved in a similar way with the help of formula (37). But one should also apply Lemma 2.1 in [6] and instead of formula (36) one should use the following expressions for the terms of the kernels of the operators in the r.h.s. of (32) and (33):

$$
\begin{aligned}
\partial_{i} \frac{e^{i \lambda \operatorname{sgn}(\operatorname{Im} \lambda)|x-y|}-1}{4 \pi|x-y| \lambda}= & \frac{i e^{i \lambda \operatorname{sgn}(\operatorname{Im} \lambda)|x-y|}\left(x_{i}-y_{i}\right)}{4 \pi|x-y|^{2}} \\
& -\frac{\left(e^{i \lambda \operatorname{sgn}(\operatorname{Im} \lambda)|x-y|}-1\right)\left(x_{i}-y_{i}\right)}{4 \pi|x-y|^{3} \lambda}
\end{aligned}
$$

The behavior of the norms of the operators (34) and (35) when $\lambda \rightarrow 0$ is controlled with the help of the formula for the kernels of the operators

$$
\partial_{i} \partial_{j} \lambda \triangle^{-1}\left(\triangle-\lambda^{2} I\right)^{-1}
$$

that is similar to (38). The fact that these norms are finite when $\lambda \rightarrow \infty$ can be proved using formula (37) and the last statement of Proposition 4

Proof of part (ii) of Theorem 6 . We have to factorize every term of the perturbation $W$ and then verify conditions (8), (9). We demonstrate how to obtain the required estimates in case of the most "singular" terms. All the other terms in (18) can be treated in a similar way using expressions (20)-(22), conditions (16), (17), part (ii) of Lemma 9] Proposition 4. Corollary 5] and Lemma 10.

First, let us consider formula (22). This term of the perturbation is already factorized. Let us consider the part $d \triangle^{-1} *$ ad $A *$ in the first term of (22). According to formula (9) one should check that the composition ad $A \triangle^{-1} \operatorname{div}(\operatorname{curl}+\lambda I)(\widehat{\triangle}-$ $\left.\lambda^{2} I\right)^{-1} d \triangle^{-1} * \operatorname{ad} A *$ is a bounded operator in $\mathcal{H}^{1}$ with the norm uniformly bounded in $\lambda$. This follows from part (31) of Lemma 10] and from (30).

Now let us consider the first term in (20), $d \triangle^{-1} * \operatorname{ad} F P_{0}$. If we write $F=$ $\sum_{a, i, j} F_{i j}^{a} T_{a} d x_{i} \wedge d x_{j}$, where $T_{a}$ is a basis of $\mathfrak{k}$, then that term is, in turn, the sum of the following ones: $d \triangle^{-1} F_{i j}^{a} * \operatorname{ad}\left(T_{a} d x_{i} \wedge d x_{j}\right) P_{0}$. Since $F_{i j}^{a}$ are real-valued functions each of these terms can be factorized as follows:

$$
\begin{aligned}
d \triangle^{-1} F_{i j}^{a} * \operatorname{ad}\left(T_{a} d x_{i}\right. & \left.\wedge d x_{j}\right) P_{0}= \\
& =\left(d \triangle^{-1} \sqrt{\left|F_{i j}^{a}\right|}\right)\left(\sqrt{\left|F_{i j}^{a}\right|} \operatorname{sgn}\left(F_{i j}^{a}\right) * \operatorname{ad}\left(T_{a} d x_{i} \wedge d x_{j}\right) P_{0}\right) .
\end{aligned}
$$


Now recall conditions (8) and (9). Let us show, for instance, that the composition $\sqrt{\left|F_{i j}^{a}\right|} \triangle^{-1} \operatorname{div}(\operatorname{curl}+\lambda I)\left(\widehat{\triangle}-\lambda^{2} I\right)^{-1} d \triangle^{-1} \sqrt{\left|F_{i j}^{a}\right|}$ is a bounded operator in $\mathcal{H}^{0}$ with the norm uniformly bounded in $\lambda$. This follows from part (31) of Lemma 10 and from the fact that by (16) the operator of multiplication by $\sqrt{\left|F_{i j}^{a}\right|}$ is bounded from $\mathcal{H}^{0, s^{\prime}}$ to $\mathcal{H}^{0, s^{\prime}+s}$ for any real $s^{\prime}$. This completes the proof of Theorem 6

\section{Scattering theory For the operators $X_{A}$ : ReduCtion From elliptic OPERATORS $S_{A}$}

In this section we show how to prove that selfadjoint extensions of symmetric operators $X_{A}$ defined in Lemma 1 are unitary equivalent. We start with the definition of these extensions.

Observe that the subspace $\operatorname{Ker} \operatorname{div}_{A}$, as well as its orthogonal complement, is an invariant subspace in $\mathcal{H}^{1}+\mathcal{H}^{0}$ for the selfadjoint operator $S_{A}$ defined in Theorem [6 The restriction of $S_{A}$ to this subspace gives an operator that is, obviously, a selfadjoint extension of the symmetric operator $X_{A}$ introduced in Lemma 1 We denote this extension by the same letter, $X_{A}:$ Ker $\operatorname{div}_{A} \rightarrow \operatorname{Ker} \operatorname{div}_{A}$.

Theorem 11. Suppose that the conditions of Theorem 6 are satisfied. Then the operators $U_{ \pm}=P_{A} W_{ \pm}\left(S_{0}, S_{A}\right) P_{0}:$ Ker div $\rightarrow$ Ker $\operatorname{div}_{A}$ are unitary and $X_{A}=$ $U_{ \pm} \operatorname{curl} U_{ \pm}^{-1}$, i.e., the operators $X_{A}$ and $X_{0}=$ curl are unitary equivalent

Proof. The key observation in the proof is that the square of the operator $S_{A}$ is diagonal in the sense that $S_{A}^{2}: \mathcal{H}^{0} \rightarrow \mathcal{H}^{0}$ and $S_{A}^{2}: \mathcal{H}^{1} \rightarrow \mathcal{H}^{1}$. Indeed, from formula (III) we immediately have

$$
S_{A}^{2}=\left(\begin{array}{cc}
P_{A} \operatorname{curl}_{A} P_{A} \operatorname{curl}_{A} P_{A}-d_{A} \operatorname{div}_{A} & 0 \\
0 & -\operatorname{div}_{A} d_{A}
\end{array}\right) .
$$

By the invariance principle for wave operators (see [12]) the wave operators $W_{ \pm}\left(S_{0}^{2}, S_{A}^{2}\right)$ for the pair $S_{0}^{2}, S_{A}^{2}$ also exist and are unitary. Moreover, we have the following formula which relates $W_{ \pm}\left(S_{0}, S_{A}\right)$ and $W_{ \pm}\left(S_{0}^{2}, S_{A}^{2}\right)$ :

$$
\begin{aligned}
& \left(W_{ \pm}\left(S_{0}, S_{A}\right)-W_{ \pm}\left(S_{0}^{2}, S_{A}^{2}\right)\right) E\left(\mathbb{R}_{+}\right)=0, \\
& \left(W_{ \pm}\left(S_{0}, S_{A}\right)-W_{\mp}\left(S_{0}^{2}, S_{A}^{2}\right)\right) E\left(\mathbb{R}_{-}\right)=0,
\end{aligned}
$$

where $E$ is the spectral measure of $S_{0}$.

Since Ker div is an invariant subspace for $S_{0}$ this subspace is also invariant for the spectral measure of $S_{0}, E\left(\mathbb{R}_{ \pm}\right):$Ker div $\rightarrow$ Ker div, and, in particular, $E\left(\mathbb{R}_{ \pm}\right)$: Ker div $\rightarrow \mathcal{H}^{1}$. But the operator $S_{A}^{2}$ is diagonal, and hence $W_{ \pm}\left(S_{0}^{2}, S_{A}^{2}\right): \mathcal{H}^{1} \rightarrow \mathcal{H}^{1}$. The last two observations together with (40) show that

$$
W_{ \pm}\left(S_{0}, S_{A}\right): \text { Ker } \operatorname{div} \rightarrow \mathcal{H}^{1} .
$$

Now using (41), the definition of the operator $S_{A}$ and the intertwining property of wave operators we have for any $\omega \in \operatorname{Ker} \operatorname{div} \bigcap \mathfrak{D}\left(S_{0}\right)$

$$
\begin{aligned}
& W_{ \pm}\left(S_{0}, S_{A}\right) S_{0}(\omega, 0)=S_{A} W_{ \pm}\left(S_{0}, S_{A}\right)(\omega, 0), \\
& W_{ \pm}\left(S_{0}, S_{A}\right)\left(P_{0} \operatorname{curl} \omega, 0\right)=S_{A}\left(W_{ \pm}\left(S_{0}, S_{A}\right) P_{0} \omega, 0\right), \\
& \left(W_{ \pm}\left(S_{0}, S_{A}\right) P_{0} \operatorname{curl} \omega, 0\right)=\left(X_{A} W_{ \pm}\left(S_{0}, S_{A}\right) P_{0} \omega, \operatorname{div}_{A} W_{ \pm}\left(S_{0}, S_{A}\right) P_{0} \omega\right) .
\end{aligned}
$$


From the last line in (42) we infer that $\operatorname{div}_{A} W_{ \pm}\left(S_{0}, S_{A}\right) P_{0} \omega=0$. Therefore

$$
\operatorname{Im} W_{ \pm}\left(S_{0}, S_{A}\right) P_{0} \subset \operatorname{Ker} \operatorname{div}_{A}
$$

and since $W_{ \pm}\left(S_{0}, S_{A}\right)$ are unitary operators we also have

$$
U_{ \pm}^{*} U_{ \pm}=P_{0} W_{ \pm}\left(S_{0}, S_{A}\right)^{*} W_{ \pm}\left(S_{0}, S_{A}\right) P_{0}=P_{0} .
$$

A calculation for $W_{ \pm}\left(S_{0}, S_{A}\right)^{*}$ similar to (42) shows that $U_{ \pm} U_{ \pm}^{*}=P_{A}$. Therefore $U_{ \pm}$are unitary operators.

Finally (43) and the last equality in (42) imply that

$$
P_{A} W_{ \pm}\left(S_{0}, S_{A}\right) P_{0} \text { curl }=X_{A} P_{A} W_{ \pm}\left(S_{0}, S_{A}\right) P_{0},
$$

or $U_{ \pm}$curl $=X_{A} U_{ \pm}$. This completes the proof of the theorem.

\section{An EXTENSION OF THE MAIN RESUlt}

In conclusion we make a few remarks on possible extensions of Theorems 6 and 11 for connections $A$ which are not smooth.

First, instead of the space $\Omega^{1}\left(\mathbb{R}^{3}, \mathfrak{k}\right)$ of smooth connection one-forms we shall consider the space $\mathcal{W}_{2, \text { loc }}^{1}\left(\mathbb{R}^{3}, \mathfrak{k}\right)$ of $\mathfrak{k}$-valued one-forms on $\mathbb{R}^{3}$ whose coefficients are elements of the Sobolev space $W_{2, l o c}^{1}\left(\mathbb{R}^{3}\right)$.

One can also define the gauge group $\mathcal{W}_{2, l o c}^{2}\left(\mathbb{R}^{3}, K\right)$ of Sobolev gauge transformations by requiring that $g \in \mathcal{W}_{2, l o c}^{2}\left(\mathbb{R}^{3}, K\right)$ if and only if $d g g^{-1} \in \mathcal{W}_{2, l o c}^{1}\left(\mathbb{R}^{3}, \mathfrak{k}\right)$ (see, for instance, [5] $)$. The space $\mathcal{W}_{2, l o c}^{2}\left(\mathbb{R}^{3}, K\right)$ is indeed a group continuously acting by gauge transformations on $\mathcal{W}_{2, l o c}^{1}\left(\mathbb{R}^{3}, \mathfrak{k}\right)$.

Obviously $\mathcal{W}_{2, l o c}^{2}\left(\mathbb{R}^{3}, K\right)$ also acts by unitary transformations on the space $\mathcal{H}^{1}$.

Now from Theorems [6 and 11 we infer the following statement.

Theorem 12. Let $A \in \mathcal{W}_{2, l o c}^{1}\left(\mathbb{R}^{3}, \mathfrak{k}\right), F=d A+\frac{1}{2}[A \wedge A]$ the curvature of $A$, where the derivatives of the coefficients of $A$ should be understood in the sense of generalized functions. There exists a positive constant $C$ such that if for some $s>3$ and $g \in \mathcal{W}_{2, l o c}^{2}\left(\mathbb{R}^{3}, K\right)$

$$
\begin{aligned}
& \left\|g \circ F\left(1+|x|^{2}\right)^{s}\right\|_{(\infty)}<C, \\
& \left\|g \circ A\left(1+|x|^{2}\right)^{\frac{s}{2}}\right\|_{(\infty)}<C,
\end{aligned}
$$

then

(i) The corresponding operator $S_{A}$ is selfadjoint with the domain $g^{-1} \mathfrak{D}\left(S_{0}\right)$.

(ii) The wave operators

$$
W_{ \pm}\left(S_{0}, S_{A}\right)=s-\lim _{t \rightarrow \pm \infty} e^{i S_{A} t} e^{-i S_{0} t}
$$

for the pair $S_{0}, S_{A}$ exist and are unitary. In particular, the selfadjoint operators $S_{0}$ and $S_{A}$ are unitary equivalent.

(iii) The operators $U_{ \pm}=P_{A} W_{ \pm}\left(S_{0}, S_{A}\right) P_{0}$ : Ker div $\rightarrow$ Ker $\operatorname{div}_{A}$ are unitary and for the operator $X_{A}=P_{A} X_{A} P_{A}$ we have $X_{A}=U_{ \pm}$curl $U_{ \pm}^{-1}$, i.e., the operators $X_{A}$ and $X_{0}$ are unitary equivalent 


\section{REFERENCES}

[1] Agmon, S., Spectral properties of Schrödinger operators and scattering theory, Ann. Scuola Norm. Sup. Pisa Cl. Sci., (4) 2 (1975), no. 2, 151-218.

[2] Birman, M. Sh., Solomyak, M. Z., $L_{2}$-theory of the Maxwell operator in arbitrary domains, Russian Math. Surveys, 42 (1987), no. 6, 75-96

[3] Birman, M. Sh., Solomyak, M. Z., The self-adjoint Maxwell operator in arbitrary domains, Algebra and Analysis, 1 (1989), no. 1, 96-110.

[4] Filonov, N., Spectral analysis of the selfadjoint operator curl in a region of finite measure, St. Petersburg Math. J., 11 (2000), 1085-1095.

[5] Jaffe, A., Taubes, C. H., Vortices and monopoles, Birkhäuser, Boston (1980).

[6] Jensen, A., Kato, T., Spectral properties of Schrödinger operators and time-decay of the wave functions, Duke Math. J., 46 (1979), 583-611.

[7] Kato, T., Wave operators and similarity for some non-selfadjoint operators, Math. Annalen, 162 (1966), 258-279.

[8] Ladyzenskaya, O. A., Uraltseva, N. N., Linear and quasilinear equations of elliptic type, Nauka, Moscow (1973).

[9] Leinfelder, H., Simander, C., Schrödinger operators with singular magnetic vector potentials, Math. Z., 176 (1981), 1-19.

[10] Picard, R., On a selfadjoint realization of curl in exterior domains, Math. Z. 229 (1998), 319-338.

[11] Reed, M., Simon, B., Methods of modern mathematical physics, vol. 2, Academic Press, London (1975).

[12] Reed, M., Simon, B., Methods of modern mathematical physics, vol. 3, Academic Press, London (1979).

[13] Reed, M., Simon, B., Methods of modern mathematical physics, vol. 4, Academic Press, London (1978).

Department of Mathematical Sciences, University of Aberdeen, Aberdeen AB24 3UE, UnITED KINGDOM

E-mail address: seva@maths.abdn.ac.uk 